\title{
On the acoustogyration diffraction of light
}

\author{
Vlokh R., Krupych O. and Martynyuk-Lototska I. \\ Institute of Physical Optics, 23 Dragomanov St., 79005 Lviv, Ukraine, \\ e-mail:vlokh@ifo.lviv.ua
}

Received: 23.05 .2007

\begin{abstract}
Interaction of acoustic and light waves with accounting of elastooptic and elastogyration effects has been theoretically described. Collinear acoustogyration (AG) diffraction in the quartz crystals has been reinvestigated and thoroughly analyzed. The AG efficiency and the AG figure of merit are calculated for those crystals and compared with the experimental results.
\end{abstract}

PACS: 78.20.Hp, 78.20.Ek

Keywords: acoustooptics, acoustogyration, piezogyration, quartz crystals

\section{Introduction}

We have shown in our preceding reports [1-3] that the propagation of ultrasonic wave in crystals, which exhibit a so-called piezogyration effect, should give rise to diffraction grating based on the modulation of antisymmetric part of dielectric permittivity, thus leading to appearance of a separate kind of diffraction of optical radiation. These phenomena have been called as acoustogyration (AG) diffraction of light, in much the same to acoustooptic (AO) diffraction. As is well known, the latter represents a common phenomenon that manifests itself in diffraction of optical radiation on the grating created through perturbation, via photoelastic coupling, of the real part of dielectric permittivity. It is interesting to remind that the analogical effect has been described and experimentally studied while recording dynamic holograms in some photorefractive crystals [4], though the reasons for creation of the diffraction grating in the latter case are photorefraction and electrogyration effects. Some important basic peculiarities of AG diffraction have been revealed and described in [1-3]. Namely, the conditions for observation of this effect in crystals of different point symmetry groups have been analyzed. Furthermore, a collinear interaction of light radiation and acoustic wave, propagated along the optic axis of optically uniaxial, gyrotropic crystals, has been found to be one of the most favourable for investigation of this effect, because the AO diffraction does not arise in this case and only AG interaction should be observable. It has also been shown that, in contrast to AO diffraction, the $\mathrm{AG}$ one can be only anisotropic, i.e. the incident and diffracted beams 
should possess orthogonal polarizations. The coefficient determining the AG figure of merit has been derived in [1-3] and the effect has been experimentally observed in quartz crystals. However, a routine mathematical analysis of the AG interaction in these works has been mainly performed, using natural analogies between the AO and AG parameters. As a consequence, the relations for AG efficiency and the figure of merit obtained in [13] reflect in general the main features of the effect but are not accurate enough in order to assess the phenomenon quantitatively. The aim of the present paper is thorough theoretical description of the phenomena mentioned above, derivation of quantitative relations for the AG diffraction efficiency and the AG figure of merit, as well as refined experimental studies of the AG diffraction in quartz crystals and comparison of the corresponding data with theoretical predictions.

\section{Experimental}

\section{a) Experimental approach}

Let us consider propagation of polarized light along the optic axis $\mathrm{Z}$ in optically uniaxial, gyrotropic crystals. Two light eigenwaves are excited in such a crystal, with right $(r)$ and left $(l)$ circular polarizations and equal intensities $\left(I_{l}=I_{r}=\frac{1}{2} E_{0}^{2}\right)$. The oscillations of the electric field vectors along the $\mathrm{X}$ and $\mathrm{Y}$ axes that correspond to the right and left circularly polarized waves are given respectively by the expressions

$$
\left\{\begin{array}{l}
E_{x}=\frac{\sqrt{2}}{2} E_{0} \cos (\omega t+\varphi) \\
E_{y}=-\frac{\sqrt{2}}{2} E_{0} \sin (\omega t+\varphi)
\end{array},\right.
$$

and

$$
\left\{\begin{array}{l}
E_{x}=\frac{\sqrt{2}}{2} E_{0} \cos [(\omega t+\varphi)+\delta] \\
E_{y}=\frac{\sqrt{2}}{2} E_{0} \sin [(\omega t+\varphi)+\delta]
\end{array} .\right.
$$

Here $\omega$ is the frequency, $\varphi$ the reference phase, $\delta$ the phase difference appearing between the right and left circularly polarized waves. The latter is caused by the optical activity effect and is determined by the difference in the phase velocities of the right and left waves or their indices of refraction $n_{r}$ and $n_{l}$ :

$$
\delta=\frac{2 \pi}{\lambda} d\left(n_{r}-n_{l}\right) .
$$

Let the geometry of anisotropic diffraction be collinear (the incident (in) and diffracted $(d)$ light waves propagate along the same direction, as well as the acoustic $(a c)$ wave, i.e. we have $K_{a c}\left\|k_{i n}\right\| k_{d}$ ) and a longitudinal acoustic wave be excited, which 
propagates along $\mathrm{Z}$ axis in the quartz crystal (the point symmetry group being 32 ). Then one can write the relation for the optical-frequency polarization in the following form:

$$
\begin{aligned}
& \Delta P_{1}^{\omega \pm \Omega}=\left(p_{1233} S_{33}^{\Omega}+i e_{123} \Delta g_{33} k_{3}\right) E_{2}^{\omega} \\
& \Delta P_{2}^{\omega \pm \Omega}=\left(p_{2133} S_{33}^{\Omega}+i e_{213} \Delta g_{33} k_{3}\right) E_{1}^{\omega},
\end{aligned}
$$

where $\Delta P_{1}^{\omega}$ and $\Delta P_{2}^{\omega}$ denote respectively the increments of $\mathrm{X}$ and $\mathrm{Y}$ components of the optical-frequency polarizations, $S_{33}^{\Omega}$ the mechanical deformation caused by acoustic wave with the frequency $\Omega, E_{1}^{\omega}$ and $E_{2}^{\omega}$ the electric field components of the incident wave, $p_{1233}$ and $p_{2133}$ the photoelastic coefficients ( $p_{1233}=p_{2133}=0$ for the quartz crystals), $k_{3}$ the light wave vector, $\Delta g_{33}$ the increment of the gyration tensor acquired due to elastogyration (ElG) effect (with $\delta_{3333}$ being a fourth-rank axial ElG tensor), $e_{123}=-e_{213}=1$ are the components of the unit Levi-Civita axial tensor. Let us now take into account that for the quartz crystals $p_{1233}=p_{2133}=0$ and $\Delta g_{33}=\delta_{3333} S_{33}^{\Omega}$. As a result, we can rewrite Eqs. (4) as

$$
\begin{aligned}
& \Delta P_{1}^{\omega}=i e_{123} \delta_{3333} S_{33}^{\Omega} k_{3} E_{2}^{\omega} \\
& \Delta P_{2}^{\omega}=-i e_{123} \delta_{3333} S_{33}^{\Omega} k_{3} E_{1}^{\omega} .
\end{aligned}
$$

As one can easily see, the anisotropic diffraction in the mentioned case can be affected only by AG interaction, while the AO interaction should not manifest itself. Moreover, the sign "minus" in the second row of Eq. (5) suggests that the diffracted radiation would possess the opposite circular polarization, when compare to the incident one. Otherwise, if the incident radiation is linearly polarized, the collinear AG diffraction would manifest itself in energy transfer between the circularly polarized eigenwaves, while the direction of energy transfer (form left wave to right one or vice versa) is determined by the sign of optical activity. This process is presented schematically in Fig. 1, using simple wave vector diagrams.

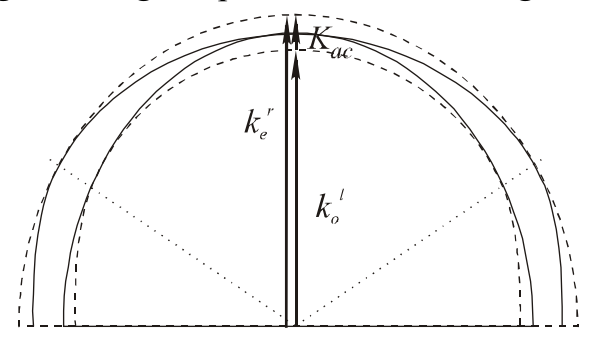

a

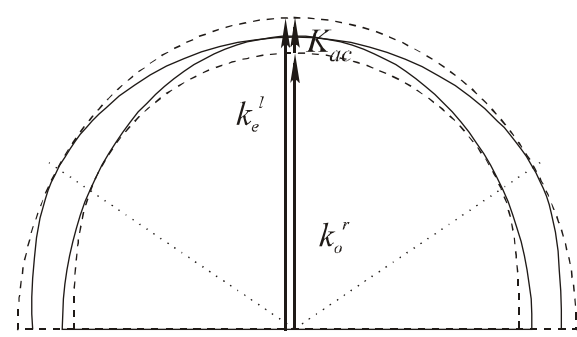

b

Fig. 1. Wave vector diagrams for the collinear AG diffraction for dextrorotatory (a) and laevorotatory (b) quartz crystals.

One can determine the diffraction efficiency as a ratio of intensities of the diffracted (e.g., left) and the incident (right) waves or as a quantity, which is mathematically 
coupled with the emergent light ellipticity. From the mentioned above it follows that there may be two different polarimetric schemes of experiment: (a) polarization the incident light has linear polarization and the emergent one is elliptical, or vice versa; (b) the incident light is circularly polarized and one should measure the intensity of diffracted light with the opposite circular. In the latter case just the ellipticity is a measure of diffraction efficiency. Accordingly, the two experimental schemes are presented in Fig. 2.

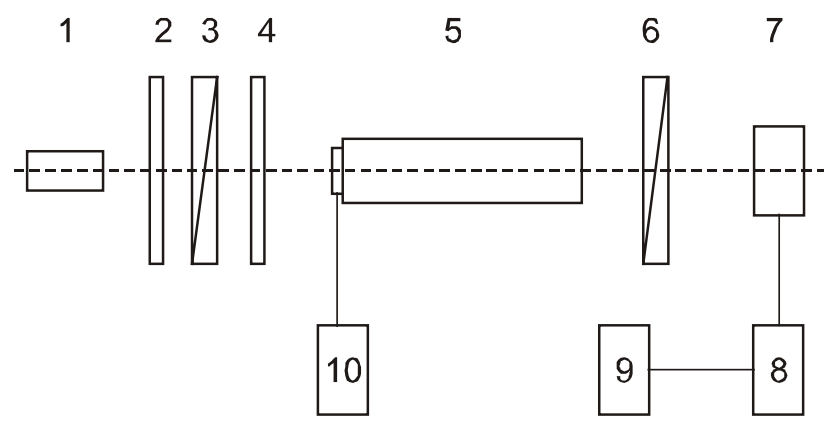

a

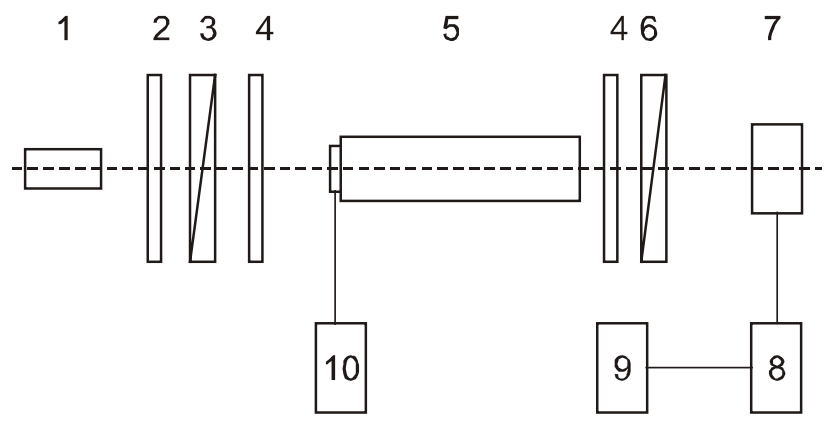

$\mathrm{b}$

Fig. 2. Two schemes used for experimental studies of the collinear AG diffraction: (a) the incident and emergent waves have elliptical and linear polarizations, respectively (the ellipticity of light or the rotation of light polarization plane are then determined in the experiment); (b) the incident light is circularly polarized (the intensity of the diffracted light with the opposite circular polarization is then determined in the experiment). $1-\mathrm{He}-\mathrm{Ne}$ laser; $2-\lambda / 4$ phase plate; 3 - polarizer, $4-\lambda / 4$ phase retardation plate; 5 - sample; 6 - analyzer; 7 - photomultiplier; 8 - lock-in amplifier; 9 - indicator; 10 - generator.

Now let us derive the relation for the AG efficiency. The intensity of diffracted circular waves may be presented as

or

$$
I_{r}^{d}=I_{r}+\Delta I=(1+\eta) I=\frac{1}{2}(1+\eta) E_{0}^{2},
$$

$$
I_{l}^{d}=I_{l}+\Delta I=(1+\eta) I=\frac{1}{2}(1+\eta) E_{0}^{2},
$$


where $\Delta I$ means the change in the intensity due to diffraction and $\eta=\Delta I / I$ the diffraction efficiency of AG interaction. Then Eqs. (1) and (2) yield in

$$
\left\{\begin{array}{l}
E_{x}=A_{r} \cos (\omega t+\varphi) \\
E_{y}=-A_{r} \sin (\omega t+\varphi)
\end{array}\right.
$$

and

$$
\left\{\begin{array}{l}
E_{x}=A_{l} \cos [(\omega t+\varphi)+\delta] \\
E_{y}=A_{l} \sin [(\omega t+\varphi)+\delta]
\end{array},\right.
$$

where

$$
\begin{aligned}
& A_{r}=\sqrt{I_{r}^{d}}=\frac{\sqrt{2}}{2} \sqrt{1+\eta} E_{0}, \\
& A_{l}=\sqrt{I_{l}^{d}}=\frac{\sqrt{2}}{2} \sqrt{1+\eta} E_{0} .
\end{aligned}
$$

The two orthogonal components of the electric field appearing owing to interference may be given by

$$
\left\{\begin{array}{l}
E_{x}=\cos (\omega t+\varphi)\left(A_{r}+A_{l} \cos \delta\right)-A_{l} \sin (\omega t+\varphi) \sin \delta \\
E_{y}=\sin (\omega t+\varphi)\left(A_{l} \cos \delta-A_{r}\right)+A_{l} \cos (\omega t+\varphi) \sin \delta
\end{array} .\right.
$$

The solutions of this system of equations with respect to $\cos (\omega t+\varphi)$ and $\sin (\omega t+\varphi)$ are

$$
\begin{aligned}
& \cos (\omega t+\varphi)=\frac{E_{x}\left(A_{l} \cos \delta-A_{r}\right)+E_{y} A_{l} \sin \delta}{A_{l}^{2}-A_{r}^{2}}, \\
& \sin (\omega t+\varphi)=\frac{E_{y}\left(A_{r}+A_{l} \cos \delta\right)-E_{x} A_{l} \sin \delta}{A_{l}^{2}-A_{r}^{2}} .
\end{aligned}
$$

Using the identity $\sin ^{2} \alpha+\cos ^{2} \alpha=1$ and Eqs. (9), we rewrite Eq. (11) as

$$
\frac{E_{x}^{2}\left[1-\sqrt{1-\eta^{2}} \cos \delta\right]}{\eta^{2} E_{0}^{2}}+\frac{E_{y}^{2}\left[1+\sqrt{1-\eta^{2}} \cos \delta\right]}{\eta^{2} E_{0}^{2}}-\frac{2 E_{x} E_{y} \sqrt{1-\eta^{2}} \sin \delta}{\eta^{2} E_{0}^{2}}=1 .
$$

In fact, this is equation of ellipse turned by an angle $\delta$ in the $X Y$ plane. This angle is defined by the optical activity of gyrotropic crystal:

$$
\delta=\frac{2 \pi}{\lambda} d\left(n_{r}-n_{l}\right)=\frac{2 \pi d}{\lambda n_{0}} G,
$$

where $n_{r}-n_{l}=\frac{G}{n_{0}}, \lambda$ is the light wavelength, $n_{0}$ - the ordinary refractive index and $G$ the scalar gyration parameter. In the eigen coordinate system Eq. (12) reduces to

$$
\frac{E_{x}^{2}\left(1-\sqrt{1-\eta^{2}}\right)}{\eta^{2} E_{0}^{2}}+\frac{E_{y}^{2}\left(1+\sqrt{1-\eta^{2}}\right)}{\eta^{2} E_{0}^{2}}=1 .
$$


It is seen that the ellipticity of emergent light is defined only by diffraction efficiency $\eta$. Thus, one can determine the AG diffraction efficiency $\eta$ provided that the emergent light ellipticity is known from experiment. In order to find this ellipticity, one can use a standard quarter-wave compensator. In this case the rotation angle of polarization plane for the light passed through the quarter-wave plate may be presented as

$$
\tan \Delta \varphi=\frac{1+\sqrt{1-\eta^{2}}}{1-\sqrt{1-\eta^{2}}} .
$$

The diffraction efficiency is then given by the relation

$$
\eta=\sqrt{1-\frac{\tan \Delta \varphi-1}{\tan \Delta \varphi+1}}
$$

\section{b) Experimental procedure}

Our experimental studies of collinear interaction of the light radiation and the acoustic wave in the quartz crystals were carried out with the aid of standard ellipsometer LEF-3M-1, which permitted measuring polarization plane rotations with the accuracy of $\Delta \varphi= \pm 1^{\prime}$. The sample of quartz crystal had the size $98 \times 26 \times 12 \mathrm{~mm}^{3}$, with the longest side oriented parallel to the optic axis ( $Z$ direction). Piezoelectric transducer was glued by epoxy glue on the $Z$ face of sample. To provide a possibility for the light to propagate through the transducer, a little hall $(\varnothing 1 \mathrm{~mm})$ was drilled in the centre of transducer plate. The piezoelectric transducer had the resonant oscillation frequency equal to $2.6 \mathrm{MHz}$. It was fabricated on the basis of PZN ceramics and excited longitudinal acoustic waves. The square of the transducer plate was equal to $8 \times 10 \mathrm{~mm}^{2}$. Notice that the resonant frequency of the transducer was far from the Bragg diffraction condition $\left(\Omega_{B}=0.66 \mathrm{MHz}\right)$. The latter may be calculated using the relation $\Omega_{B}=\frac{G v_{33}}{\lambda n_{0}}$, where $v_{33}=6320 \mathrm{~m} / \mathrm{s}$ is the velocity of longitudinal acoustic wave and $\lambda=632.8 \mathrm{~nm}$ is the optical wavelength. The divergence of acoustic wave was estimated using the relation $\gamma=\arcsin \frac{1.22 \Lambda}{D}$ ( $D \approx 9 \mathrm{~mm}$ being the linear dimension of the transducer and $\Lambda=2.4 \times 10^{-3} \mathrm{~m}$ the acoustic wavelength) and was approximately equal to $\gamma \simeq 19^{\circ}$. This means that the acoustic waves reflected from the front and back faces of sample and its lateral faces can interfere with the onward wave, leading to appearance of spatial harmonics of the acoustic field. Moreover, such a divergent acoustic wave includes the acoustic wave vectors, which deviate from the direction of optic axis by $\gamma \simeq 19^{\circ}$. This deviation may lead to appearance of small-angular anisotropic AO diffraction, which could behave similar to the $A G$ one. In order to prevent reflections of acoustic wave from the lateral and opposite faces, we used so-called dampers. These were prepared from organic-glass plates having 
one side polished and the other ribbed. The dampers were adjusted to the sample faces from the polished side, using oil as an intermediate layer for reaching acoustic matching. Thus, the acoustic wave moved out of sample through the boundary between the sample and organic-glass plates and then scattered by the ribbed opposite side of the glass.

In our experiments we used mainly the scheme (a) shown in Fig. 2. This scheme actually corresponds to the well-known Senarmont method for measuring the light ellipticity. The scheme (b) was employed only for checking the experimental results. The ellipticity caused by AG interaction was strictly measured by rotating polarizer 3 until the intensity of light passed through the analyzer 6 was compensated. Prior to measurements of acoustically induced changes in the optical properties of crystals under test, their natural optical rotation was compensated by rotating the polarizer in a proper manner.

\section{Experimental results}

As one can see from Fig. 3, applying of acoustic signal leads to well visible optical transmission of our system, thus evidencing that the acoustic wave affects the optical properties of quartz crystals. We have measured the frequency dependence of acoustically induced polarization plane rotation $\Delta \varphi$ and the intensity of transmitted light at the constant level of acoustic signal (the root-mean-square voltage amplitude is $10 \mathrm{~V}$ ). These dependences are presented in Fig. 4. One can see that the frequency dependence of the intensity of diffracted beam does not agree with the same dependence of angular rotation of polarization plane, though the both parameters should correspond to the frequency dependence of the diffraction efficiency. Notice that no dampers have been used in the mentioned case. Thus, it would be reasonable to assume that relatively large optical transmission is not associated with the AG but is caused, probably, by a divergent acoustic wave and the corresponding AO interaction.

Let us check if we have indeed used the scheme (b) (see Fig. 2). The technique for checking is based on the fact that the AG for right-handed crystals should manifest itself in the energy transfer from the right to left circular wave but not from the left to right (see

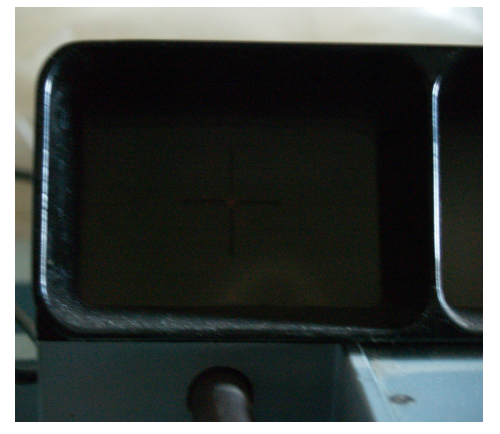

(a)

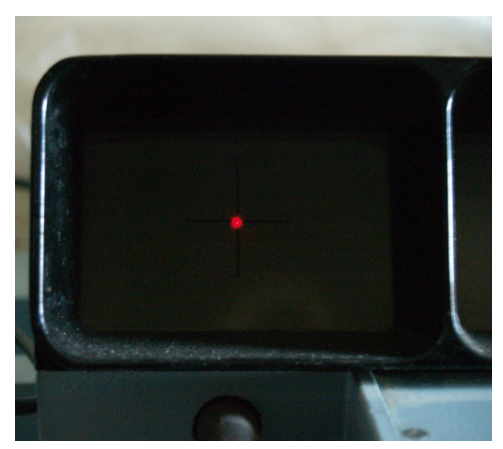

(b)

Fig. 3. Photograph of diffraction in the quartz crystals: (a) without any acoustic signal applied and (b) with the acoustic signal $(\Omega=2.6 \mathrm{MHz}$ and the root-mean-square voltage amplitude $10 \mathrm{~V}$ ) applied to the crystal. 


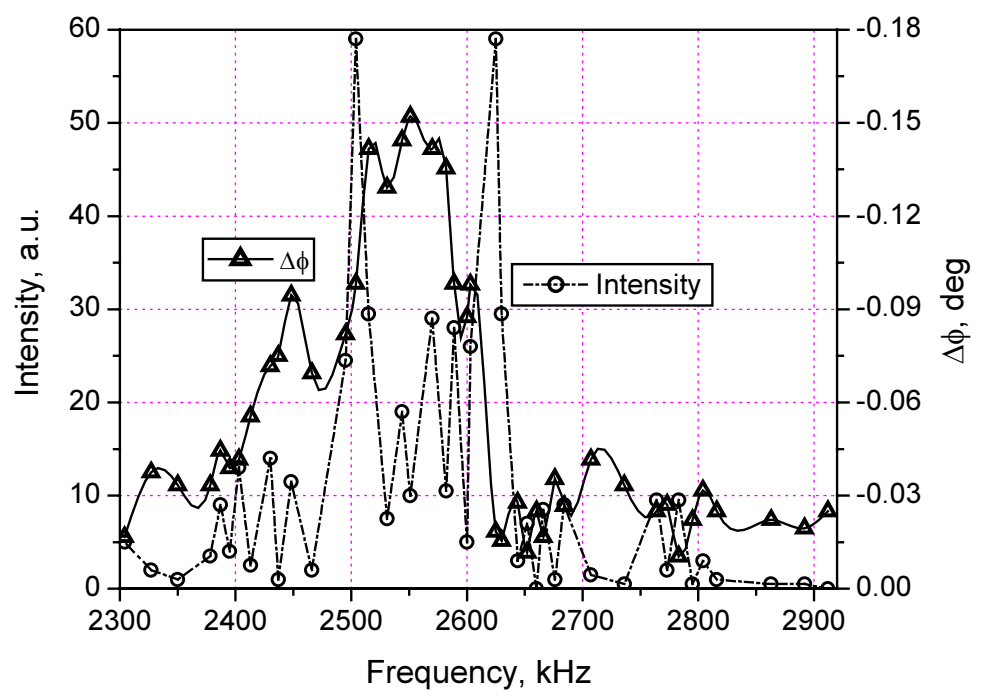

Fig. 4. Dependence of polarization plane rotation angle $\Delta \varphi$ (triangles) and intensity of the diffracted light (circles) on the frequency of acoustic signal.

Fig. 1). As a result, if the sign of circular polarization of the incident light is changed, the AG diffraction should exist in one case and contrary for the alternative case. It has been found experimentally that some kind of diffraction exists in the both cases. This fact suggests that the phenomena observed by us stem from a parasitic $\mathrm{AO}$ interaction rather than the AG effect.

To eliminate the influence of the parasitic AO diffraction, which is in fact "quasicollinear", we have used the dampers described above. The results of our studies are presented in Fig. 5. It is clear that utilization of the lateral dampers leads to appearance of

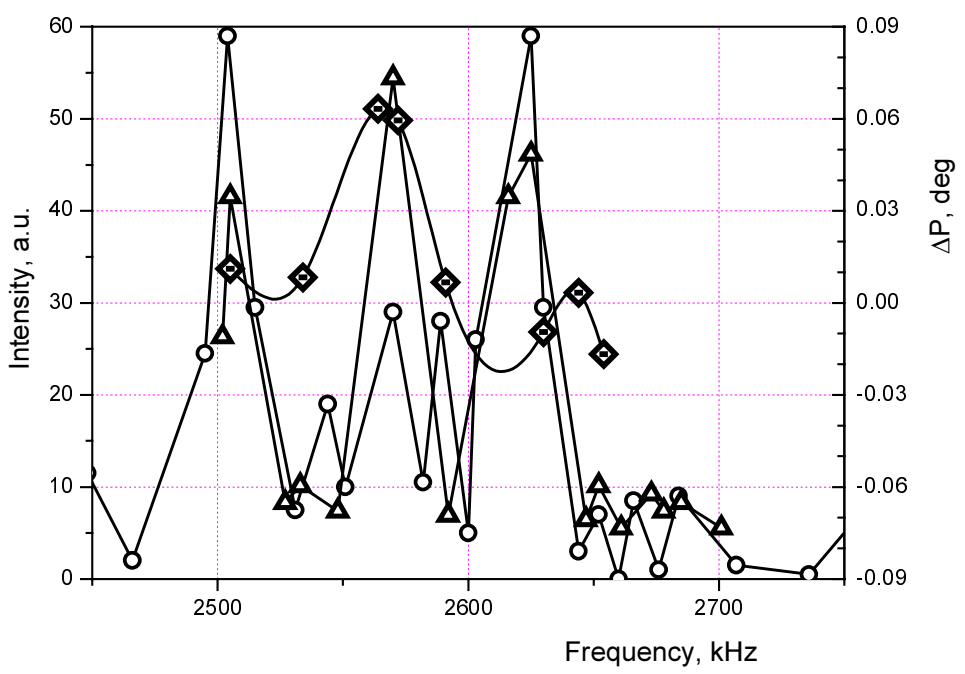

Fig. 5. Dependences of intensity of the diffracted beam (triangles) and polarization plane rotation (diamonds) on the frequency of acoustic signal. Dependence of measured intensity of the diffracted beam obtained without dampers is presented by circles (the root-mean-square voltage amplitude is $10 \mathrm{~V}$ ). 
a peak at the central (resonant) frequency and essential decreasing of the peak of polarization plane rotation. Adding of the damper to the opposite $\mathrm{Z}$ face of the sample does not change essentially the value of the polarization plane rotation (see Fig. 6). Nonetheless, we have found that a drift of extinction position is observed in the vicinity of transducer resonant frequency occurs if the acoustic signal is applied. Switching-off of the acoustic signal results in a long temporal relaxation of the rotation angle of polarization plane and approximately 7-10 minutes are needed for the polarization azimuth to return to its initial position. We have supposed that the transducer heats the sample at the resonant frequency. The change in the sample temperature occurred in the vicinity of transducer has been measured by a thermocouple. One can see from Fig. 6 that the frequency behaviour of this temperature change is similar to the same dependence of the rotation of polarization plane and the maximal change in the temperature at the resonant frequency is approximately equal to $0.8 \mathrm{~K}$. It is necessary to stress that such a temperature change is enough for rotating the polarization plane by the angle of $0.06 \mathrm{deg}$, as a result of thermogyration effect in the quartz crystals [5]. Thus, one can suppose that the observed diffraction intensity is caused by parasitic AO diffraction, which is related to divergence of acoustic beam and its multiple reflections at the interference, while the rotation of polarization plane is caused by heating of sample and a subsequent thermogyration effect.

\section{Theoretical description of AG interaction}

Now we need relations for the AG diffraction efficiency taking the acoustic wave power and the constitutive coefficients of our material into consideration. Let us derive those relations. The optical-frequency dielectric impermeability of gyrotropic crystals for the case of light propagation in the $\mathrm{Z}$ direction may be written as [6]

$$
\begin{gathered}
B_{1,2}=\frac{1}{\varepsilon_{1,2}}=\left(\frac{1}{n_{1,2}^{2}}\right)= \\
\tilde{g}_{33}^{2}=g_{33}^{2} / \bar{n}^{10}, g_{k n}^{0}=\beta_{k n m l} \sigma_{m l}=\delta_{k n m l} S_{m l},
\end{gathered}
$$

where $\varepsilon_{1,2}$ are the dielectric constants, $n_{1,2}$ the refractive indices, $\bar{n}$ the mean refractive index, $\beta_{k n m l}$ the piezogyration tensor and $\sigma_{m l}$ the mechanical stresses.

The dielectric permittivity of acoustically non-perturbed gyrotropic crystal $\varepsilon_{1,2}^{0}$ reads as

$$
\varepsilon_{1,2}^{0}=\frac{2}{B_{11}^{0}+B_{22}^{0} \pm \sqrt{\left(B_{11}^{0}-B_{22}^{0}\right)^{2}+4 g_{33}^{2} / \bar{n}^{8}}},
$$

while the dielectric permittivity $\varepsilon_{1,2}^{p}$ of crystal perturbed by the mechanical deformations of acoustic wave may be presented in the form 
Vlokh R. et al
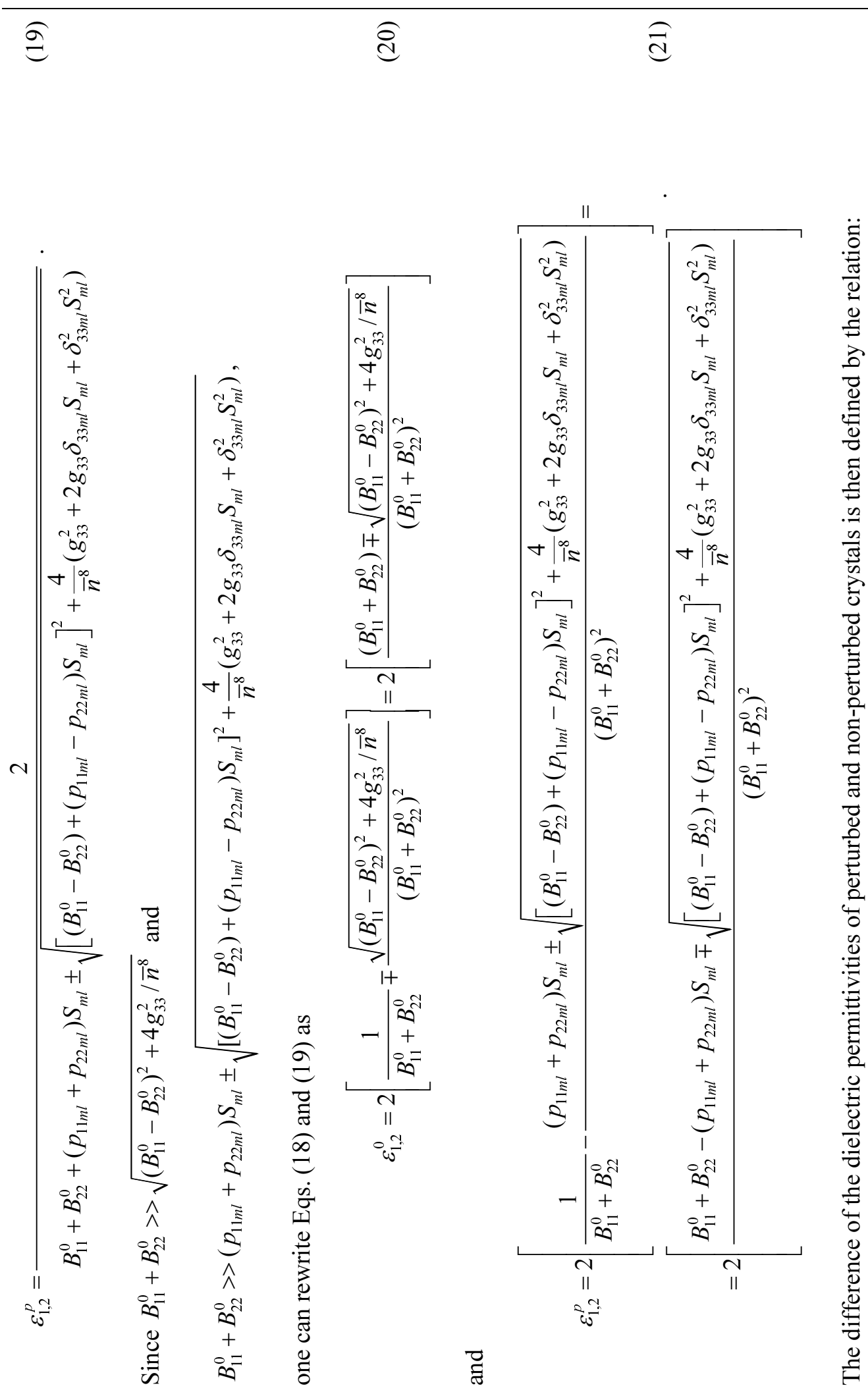


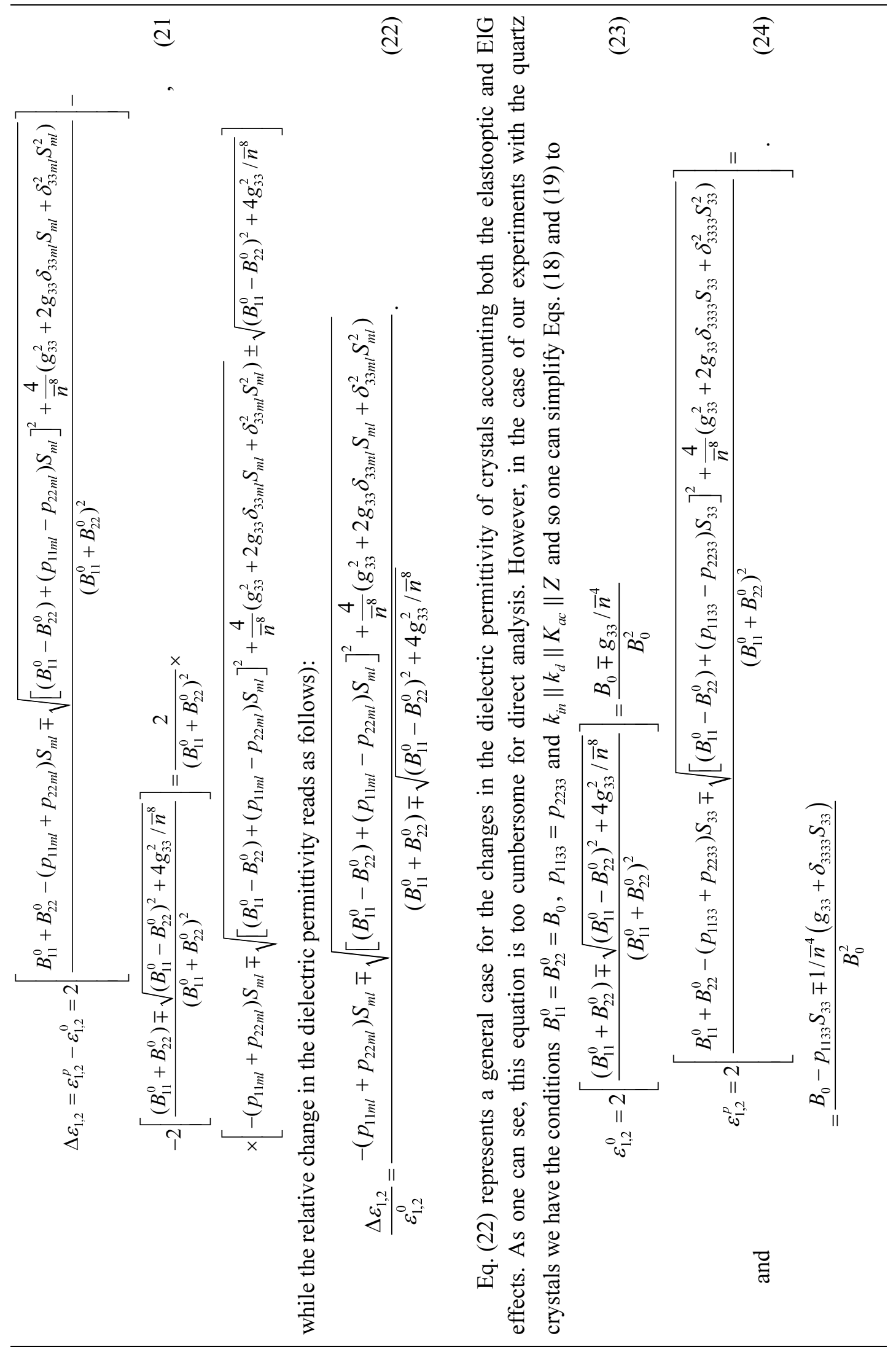


Then we obtain

$$
\Delta \varepsilon_{1,2}=\frac{-p_{1133} S_{m l} \mp 1 / \bar{n}^{4}\left(g_{33}+\delta_{3333} S_{33}\right) \pm g_{33} / \bar{n}^{4}}{B_{0}^{2}}
$$

and

$$
\frac{\Delta \varepsilon_{1,2}}{\varepsilon_{0}}=\frac{-\bar{n}^{4} p_{1133} S_{33} \mp \delta_{3333} S_{33}}{a_{0} \bar{n}^{4} \mp g_{33}}=\frac{-\bar{n}^{4} p_{1133} S_{33}}{\bar{n}^{2} \mp g_{33}} \mp \frac{\delta_{3333} S_{33}}{\bar{n}^{2} \mp g_{33}} .
$$

One can neglect in Eq. (26) the terms including $g_{33}$, since $g_{33}<<\bar{n}^{2}$. Therefore we arrive at the relation

$$
\frac{\Delta \varepsilon_{1,2}}{\varepsilon_{0}}=-\bar{n}^{2} p_{1133} S_{m l} \mp \frac{\delta_{3333} S_{33}}{\bar{n}^{2}} .
$$

The diffraction efficiency of interaction of the light radiation and the acoustic wave for the case of our experiment should be defined only by the second term in Eq. (27) (see Eq. (5) for explanation). Following [7], we write the relation for the diffraction efficiency as

$$
\eta=\frac{I}{I_{0}}=\sin ^{2}\left[\frac{\pi n L}{2 \lambda_{0} \cos \theta_{B}} \frac{\Delta \varepsilon}{\varepsilon_{0}}\right],
$$

where $L$ represents the length of interaction of acoustic and optical wave, $\theta_{B}$ the Bragg angle and $\lambda_{0}$ the wavelength of optical radiation. In our case $\theta_{B}=0, \cos \theta_{B}=1$ and $S_{33}=\sqrt{\frac{2 P_{a}}{\rho v_{33}^{3} L H}}$, with $P_{a}$ being the acoustic wave power, $\rho$ the density of crystal, $v_{33}$ the acoustic wave velocity and $H$ the width of the acoustic beam. Hence, we get

$$
\eta=\sin ^{2}\left[\frac{\pi n L}{2 \lambda_{0}} \frac{\delta_{k n 33} S_{33}}{n^{2}}\right]=\sin ^{2}\left[\frac{\pi}{2 \lambda_{0}} \sqrt{\frac{2 \delta_{3333}^{2} P_{a} L}{\rho v_{33}^{3} n^{2} H}}\right] .
$$

Following a common definition of AO figure of merit, one can introduce analogical parameter for description of the AG interaction. The corresponding AG figure of merit is given by

$$
M_{a g}=\frac{\delta_{k n 33}^{2}}{\rho v_{33}^{3} n^{2}} .
$$

It is necessary to note that Eq. (30) is exactly the same is it was obtained in [1-3]. The AG diffraction efficiency may be presented as

$$
\eta=\sin ^{2}\left[\frac{\pi}{2 \lambda_{0}} \sqrt{2 M_{a g} P_{a} \frac{L}{H}}\right],
$$

or 


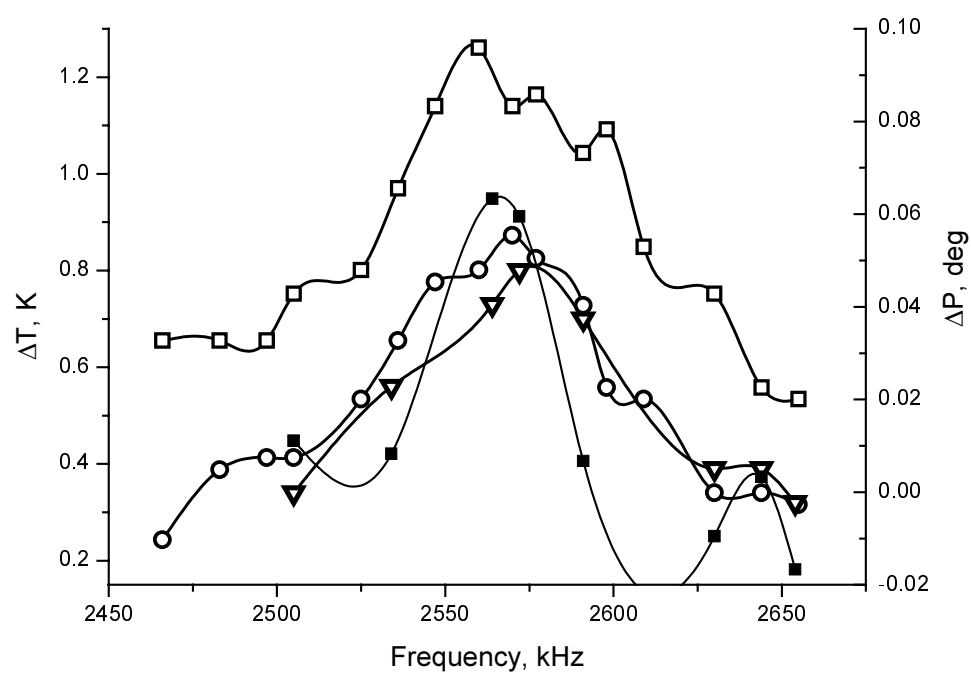

Fig. 6. Frequency dependence of the change in sample temperature occurred in the spatial vicinity of glued piezoelectric transducer when the signal with the root-mean-square voltage amplitude $10 \mathrm{~V}$ is applied: open squares - sample without dampers, open circles - sample with the lateral dampers, and open triangles - sample with the lateral and Z-opposite face dampers. Frequency dependence of the angle of polarization plane rotation is depicted with full squares.

$$
\eta \approx \frac{\pi^{2}}{2 \lambda_{0}^{2}} M_{a g} P_{a} \frac{L}{H}
$$

Using the piezogyration coefficient determined for the quartz crystals $\left(\beta_{33}=1.5 \times 10^{-13} \mathrm{~m}^{2} / \mathrm{N}[8]\right)$ and the other necessary parameters $\left(C_{33}=105 \times 10^{9} \mathrm{~N} / \mathrm{m}^{2}\right.$, $v_{33}=6320 \mathrm{~m} / \mathrm{s}, \rho=2650 \mathrm{~kg} / \mathrm{m}^{3}, n=1.5428, g_{33}=10^{-4}$ and $p_{13}=0.26$ [5]), one can calculate the ElG coefficient $\left(\delta_{33}=1.6 \times 10^{-2}\right.$ ) and, finally, the AG figure of merit for the quartz crystals $\left(M_{a g}=1.6 \times 10^{-19} \mathrm{~s}^{3} / \mathrm{kg}\right)$.

The diffraction efficiency is defined by the rotation angle of polarization plane $\left(\Delta \varphi \leq 0.06^{\circ}\right.$ ) and Eq. (16). On the basis of our experimental results, it is equal to $\eta \approx 4.5 \times 10^{-2}$. However, in order to reach such the value of diffraction efficiency, the power of acoustic wave should be $P_{a} \geq 230 \mathrm{~W}$, while in our experiments this value does not exceed $\approx 0.04 \mathrm{~W}$. Thus, the acoustic power and the ElG coefficient typical for the quartz crystals are not high enough for observation of AG diffraction of light.

However, there exists another possibility for observation of these phenomena. Achieving the necessary deformations driven by acoustic waves is not an easy problem, but these deformations can naturally exist in ferroelastic materials. On the other side, ferroelastic domains can be optical active and so enantiomorphous [9]. Then the gyration will be spatially modulated by spontaneous deformation owing to piezogyration effect in multidomain ferroelastic crystals. The effect of diffraction of optical radiation on such 
domain structure has been revealed by as in $\mathrm{Ba}_{2} \mathrm{NaNb}_{5} \mathrm{O}_{15}$ crystals [10] and the ElG component (or the AG one for the standing wave) has been separated. Moreover, the coefficients of ElG and photoelastic matrices estimated on the basis of diffraction efficiency have been comparable with those for the other materials.

\section{Conclusion}

In the present paper we have derived the relations describing the AG diffraction of light, following from the basic equations for dielectric impermeability. The diffraction efficiency and the AG figure of merit have been shown to depend on ElG coefficients of materials. The experimental studies of AG diffraction in the collinear scheme performed for the quartz crystals show that a lot of parasitic factors could affect the final experimental results. These are a divergence and multiple reflections of the acoustic beam, heating of sample by the transducer, etc. Nevertheless, the AG effect is too small to be observed in the quartz crystals because of a smallness of ElG parameter and high ultrasonic wave velocity. On the other side, this effect has already been observed in the conditions of light diffraction on enantiomorphous ferroelastic domain structure and a standing wave of the modulated gyration.

\section{Acknowledgement}

The authors acknowledge financial support of this study from the Ministry of Education and Science of Ukraine (the Project N0106U000616).

\section{References}

1. Vlokh RO, Pyatak YuA and Skab IP, 1991. Collinear acoustogyration interaction of light and ultrasonic. Rep. Ukrain. Acad. Sci. 7: 39-41.

2. Vlokh RO, Pyatak YuA and Skab IP, 1989. Acoustogyration diffraction of light in quartz crystals. Izv. AN SSSR, Ser. Fiz. 53: 1339-1341.

3. Vlokh R, Vlokh O, Pyatak Yu and Skab I, 1990. Acoustogyration diffraction of light. Ferroelectrics. 110: 1117-1121.

4. Khitrova G, Rouède D, Kukhtarev NV and Gibbs HM, 1989. New nonphotorefractive mechanism for two-beam coupling in a crystallographic-cut photorefractive crystal. Phys. Rev. Lett. 62: 1110-1113.

5. Shaskolskaya M.P. Acoustic crystals. Moscow: Nauka (1982).

6. Vlokh OG and Kobylyanskii VB, 1974. The influence of parameters of gyrotropic crystals on the behaviour of light polarization. Ukr. J. Phys. 19: 40-46.

7. Balakshii V.I., Parygin V.N. and Chirkov L.E. Physical fundamentals of acoustooptics. Moscow: Izdatel'stvo Radio i Sviaz' (1985).

8. Vlokh R, Pyatak Yu and Skab I, 1991. Piezogyration in enantiomorphous quartz crystals. Opt. Spectr. 70: 243-244. 
9. Vlokh RO and Slivka VY, 1989. Enantiomorphism of ferroelastic domains. Ferroelectrics. 98: $167-169$.

10. Vlokh R, Gotra Z, Pyatak Yu and Skab I, 1994. Elastooptic and elastogyration light diffraction by the domain structure of $\mathrm{Ba}_{2} \mathrm{NaNb}_{5} \mathrm{O}_{15}$ crystals. Ferroelectrics. 157: $147-152$. 